\title{
The Relationship Between Propagation Characteristics and Nonlinearity of Cryptographic Functions
}

\author{
Jennifer Seberry \\ (The University of Wollongong, Wollongong, NSW 2522, Australia \\ jennie@cs.uow.edu.au) \\ Xian-Mo Zhang \\ (The University of Wollongong, Wollongong, NSW 2522, Australia \\ xianmo@cs.uow.edu.au) \\ Yuliang Zheng \\ (Monash University, Melbourne, VIC 3199, Australia \\ yzheng@fcit.monash.edu.au)
}

\begin{abstract}
The connections among the various nonlinearity criteria is currently an important topic in the area of designing and analyzing cryptographic functions. In this paper we show a quantitative relationship between propagation characteristics and nonlinearity, two critical indicators of the cryptographic strength of a Boolean function. We also present a tight lower bound on the nonlinearity of a cryptographic function that has propagation characteristics.
\end{abstract}

Key Words: Cryptography, Boolean functions, Encryption functions, Nonlinearity, Propagation Characteristics, SAC, S-boxes.

Category: E.3

\section{Introduction}

Data Encryption Standard or DES is a cryptographic algorithm most widely used by industrial, financial and commercial sectors all over the world [NBS77]. DES is also the root of many other data encryption algorithms proposed in the past decade, including LOKI [BKPS93], FEAL [Miy91] and IDEA [LM91, LaSM91, Lai92]. A core component of these encryption algorithms are the socalled S-boxes or substitution boxes, each essentially a tuple of nonlinear Boolean functions. In most cases, these boxes are the only nonlinear component in an underlying encryption algorithm. The same can be said with one-way hashing algorithms which are commonly employed in the process of signing and authenticating electronic messages [ZPS93, Riv92, NIST93]. These all indicate the vital importance of the design and analysis of nonlinear cryptographic Boolean functions.

Encryption and authentication require cryptographic (Boolean) functions with a number of critical properties that distinguish them from linear (or affine) functions. Among these properties are high nonlinearity, high degree of propagation, few linear structures, high algebraic degree etc. These properties are often called nonlinearity criteria. An important topic is to investigate relationships among the various nonlinearity criteria. Progress in this direction has been made 
in [SZZ94d], where connections have been revealed among the strict avalanche characteristic, differential characteristics, linear structures and nonlinearity, of quadratic functions.

In this paper we carry on the investigation initiated in [SZZ94d] and bring together nonlinearity and propagation characteristic of a function (quadratic or non-quadratic). These two cryptographic criteria are seemly quite separate, in the sense that the former indicates the minimum distance between a Boolean function and all the affine functions whereas the latter forecasts the avalanche behavior of the function when some input bits to the function are complemented.

In particular we show that if $f$, a function on $V_{n}$, satisfies the propagation criterion with respect to all but a subset $\Re$ of $V_{n}$, then the nonlinearity of $f$ satisfies $N_{f} \geq 2^{n-1}-2^{n-\frac{1}{2} \rho-1}$, where $\rho$ is the maximum dimension a linear sub-space contained in $\{0\} \cup\left(V_{n}-\Re\right)$ can achieve.

We also show that $2^{n-2}$ is the tight lower bound on the nonlinearity of $f$ if $f$ satisfies the propagation criterion with respect to at least one vector in $V_{n}$. As an immediate consequence, the nonlinearity of a function that fulfills the SAC or strict avalanche criterion is at least $2^{n-2}$.

Two techniques are employed in the proofs of our main results. The first technique is in regard to the structure of $\Re$, the set of vectors where the function $f$ does not satisfy the propagation criterion. By considering a linear sub-space with the maximum dimension contained in $\{0\} \cup\left(V_{n}-\Re\right)$, together with its complementary sub-space, we will be able to identify how the vectors in $\Re$ are distributed. The second technique is based on a novel idea of refining Parseval's equation, a well-known relationship in the theory of orthogonal transforms. A combination of these two techniques together with some careful analyses proves to be a powerful tool in examining the relationship among nonlinearity criteria.

The organization of the rest of the paper is as follows: Section 2 introduces basic notations and conventions, while Section 3 presents background information on the Walsh-Hadamard transform. The distribution of vectors where the propagation criterion is not satisfied is discussed in Section 4. This result is employed in Section 5 where a quantitative relationship between nonlinearity and propagation characteristics is derived. This relationship is further developed in Section 6 to identify a tight lower bound on nonlinearity of functions with propagation characteristics. The paper is closed by some concluding remarks in Section 7.

\section{Basic Definitions}

We consider Boolean functions from $V_{n}$ to $G F(2)$ (or simply functions on $V_{n}$ ), $V_{n}$ is the vector space of $n$ tuples of elements from $G F(2)$. The truth table of a function $f$ on $V_{n}$ is a $(0,1)$-sequence defined by $\left(f\left(\alpha_{0}\right), f\left(\alpha_{1}\right), \ldots, f\left(\alpha_{2^{n}-1}\right)\right)$, and the sequence of $f$ is a $(1,-1)$-sequence defined by $\left((-1)^{f\left(\alpha_{0}\right)},(-1)^{f\left(\alpha_{1}\right)}\right.$, $\left.\ldots,(-1)^{f\left(\alpha_{2} n-1\right)}\right)$, where $\alpha_{0}=(0, \ldots, 0,0), \alpha_{1}=(0, \ldots, 0,1), \ldots, \alpha_{2^{n-1}-1}=$ $(1, \ldots, 1,1)$. The matrix of $f$ is a $(1,-1)$-matrix of order $2^{n}$ defined by $M=$ $\left((-1)^{f\left(\alpha_{i} \oplus \alpha_{j}\right)}\right)$. $f$ is said to be balanced if its truth table contains an equal number of ones and zeros.

An affine function $f$ on $V_{n}$ is a function that takes the form of $f\left(x_{1}, \ldots, x_{n}\right)=$ $a_{1} x_{1} \oplus \cdots \oplus a_{n} x_{n} \oplus c$, where $a_{j}, c \in G F(2), j=1,2, \ldots, n$. Furthermore $f$ is called a linear function if $c=0$. 
Definition 1. The Hamming weight of a $(0,1)$-sequence $s$, denoted by $W(s)$, is the number of ones in the sequence. Given two functions $f$ and $g$ on $V_{n}$, the Hamming distance $d(f, g)$ between them is defined as the Hamming weight of the truth table of $f(x) \oplus g(x)$, where $x=\left(x_{1}, \ldots, x_{n}\right)$. The nonlinearity of $f$, denoted by $N_{f}$, is the minimal Hamming distance between $f$ and all affine functions on $V_{n}$, i.e., $N_{f}=\min _{i=1,2, \ldots, 2^{n+1}} d\left(f, \varphi_{i}\right)$ where $\varphi_{1}, \varphi_{2}, \ldots, \varphi_{2^{n+1}}$ are all the affine functions on $V_{n}$.

Note that the maximum nonlinearity of functions on $V_{n}$ coincides with the covering radius of the first order binary Reed-Muller code $R M(1, n)$ of length $2^{n}$, which is bounded from above by $2^{n-1}-2^{\frac{1}{2} n-1}$ (see for instance [CKHFMS85]). Hence $N_{f} \leq 2^{n-1}-2^{\frac{1}{2} n-1}$ for any function on $V_{n}$. Next we introduce the definition of propagation criterion.

Definition 2. Let $f$ be a function on $V_{n}$. We say that $f$ satisfies

1. the propagation criterion with respect to $\alpha$ if $f(x) \oplus f(x \oplus \alpha)$ is a balanced function, where $x=\left(x_{1}, \ldots, x_{n}\right)$ and $\alpha$ is a vector in $V_{n}$.

2. the propagation criterion of degree $k$ if it satisfies the propagation criterion with respect to all $\alpha \in V_{n}$ with $1 \leq W(\alpha) \leq k$.

$f(x) \oplus f(x \oplus \alpha)$ is also called the directional derivative of $f$ in the direction $\alpha$. The above definition for propagation criterion is from $\left[\mathrm{PLL}^{+} 91\right]$. Further work on the topic can be found in [PGV91]. Note that the strict avalanche criterion (SAC) introduced by Webster and Tavares [Web85, WT86] is equivalent to the propagation criterion of degree 1 and that the perfect nonlinearity studied by Meier and Staffelbach [MS90] is equivalent to the propagation criterion of degree $n$ where $n$ is the number of the coordinates of the function.

While the propagation characteristic measures the avalanche effect of a function, the linear structure is a concept that in a sense complements the former, namely, it indicates the straightness of a function.

Definition 3. Let $f$ be a function on $V_{n}$. A vector $\alpha \in V_{n}$ is called a linear structure of $f$ if $f(x) \oplus f(x \oplus \alpha)$ is a constant.

By definition, the zero vector in $V_{n}$ is a linear structure of all functions on $V_{n}$. It is not hard to see that the linear structures of a function $f$ form a linear sub-space of $V_{n}$. The dimension of the sub-space is called the linearity dimension of $f$. We note that it was Evertse who first introduced the notion of linear structure (in a sense broader than ours) and studied its implication on the security of encryption algorithms [Eve88].

A $(1,-1)$-matrix $H$ of order $m$ is called a Hadamard matrix if $H H^{t}=m I_{m}$, where $H^{t}$ is the transpose of $H$ and $I_{m}$ is the identity matrix of order $m$. A Sylvester-Hadamard matrix of order $2^{n}$, denoted by $H_{n}$, is generated by the following recursive relation

$$
H_{0}=1, H_{n}=\left[\begin{array}{cc}
H_{n-1} & H_{n-1} \\
H_{n-1} & -H_{n-1}
\end{array}\right], n=1,2, \ldots
$$

Let $\ell_{i}, 0 \leq i \leq 2^{n}-1$, be the $i$ row of $H_{n}$. By Lemma 2 of [SZZ94a], $\ell_{i}$ is the sequence of a linear function $\varphi_{i}(x)$ defined by the scalar product $\varphi_{i}(x)=\left\langle\alpha_{i}, x\right\rangle$, where $\alpha_{i}$ is the $i$ th vector in $V_{n}$ according to the ascending order. 
Definition 4. Let $f$ be a function on $V_{n}$. The Walsh-Hadamard transform of $f$ is defined as

$$
\hat{f}(\alpha)=2^{-\frac{n}{2}} \sum_{x \in V_{n}}(-1)^{f(x) \oplus\langle\alpha, x\rangle}
$$

where $\alpha=\left(a_{1}, \ldots, a_{n}\right) \in V_{n}, x=\left(x_{1}, \ldots, x_{n}\right),\langle\alpha, x\rangle$ is the scalar product of $\alpha$ and $x$, namely, $\langle\alpha, x\rangle=\bigoplus_{i=1}^{n} a_{i} x_{i}$, and $f(x) \oplus\langle\alpha, x\rangle$ is regarded as a real-valued function.

The Walsh-Hadamard transform, also called the discrete Fourier transform, has numerous applications in areas ranging from physical science to communications engineering. It appears in several slightly different forms [Rot76, MS77, Dil72]. The above definition follows the line in [Rot76]. It can be equivalently written as

$$
\left(\hat{f}\left(\alpha_{0}\right), \hat{f}\left(\alpha_{1}\right), \ldots, \hat{f}\left(\alpha_{2^{n}-1}\right)\right)=2^{-\frac{n}{2}} \xi H_{n}
$$

where $\alpha_{i}$ is the ith vector in $V_{n}$ according to the ascending order, $\xi$ is the sequence of $f$ and $H_{n}$ is the Sylvester-Hadamard matrix of order $2^{n}$.

Definition 5. A function $f$ on $V_{n}$ is called a bent function if its Walsh-Hadamard transform satisfies

$$
\hat{f}(\alpha)= \pm 1
$$

for all $\alpha \in V_{n}$.

Bent functions can be characterized in various ways [AT90, Dil72, SZZ94a, YH89]. In particular the following four statements are equivalent:

(i) $f$ is bent.

(ii) $\langle\xi, \ell\rangle= \pm 2^{\frac{1}{2} n}$ for any affine sequence $\ell$ of length $2^{n}$, where $\xi$ is the sequence of $f$.

(iii) $f$ satisfies the propagation criterion with respect to all non-zero vectors in $V_{n}$.

(iv) $M$, the matrix of $f$, is a Hadamard matrix.

Bent functions on $V_{n}$ exist only when $n$ is even [Rot76]. Another important property of bent functions is that they achieve the highest possible nonlinearity $2^{n-1}-2^{\frac{1}{2} n-1}$.

\section{More on Walsh-Hadamard transform and Nonlinearity}

As the Walsh-Hadamard transform plays a key role in the proofs of main results to be described in the following sections, this section provides some background knowledge on the transform. More information regarding the transform can be found in [MS77, Dil72]. In addition, Beauchamp's book [Bea84] is a good source of information on other related orthogonal transforms with their applications.

Given two sequences $a=\left(a_{1}, \ldots, a_{m}\right)$ and $b=\left(b_{1}, \ldots, b_{m}\right)$, their componentwise product is defined by $a * b=\left(a_{1} b_{1}, \ldots, a_{m} b_{m}\right)$. Let $f$ be a function on $V_{n}$. For a vector $\alpha \in V_{n}$, denote by $\xi(\alpha)$ the sequence of $f(x \oplus \alpha)$. Thus $\xi(0)$ is the sequence of $f$ itself and $\xi(0) * \xi(\alpha)$ is the sequence of $f(x) \oplus f(x \oplus \alpha)$. 
Set

$$
\Delta(\alpha)=\langle\xi(0), \xi(\alpha)\rangle,
$$

the scalar product of $\xi(0)$ and $\xi(\alpha) . \Delta(\alpha)$ is also called the auto-correlation of $f$ with a shift $\alpha$. Obviously, $\Delta(\alpha)=0$ if and only if $f(x) \oplus f(x \oplus \alpha)$ is balanced, i.e., $f$ satisfies the propagation criterion with respect to $\alpha$. On the other hand, if $|\Delta(\alpha)|=2^{n}$, then $f(x) \oplus f(x \oplus \alpha)$ is a constant and hence $\alpha$ is a linear structure of $f$.

Let $M=\left((-1)^{f\left(\alpha_{i} \oplus \alpha_{3}\right)}\right)$ be the matrix of $f$ and $\xi$ be the sequence of $f$. Due to a very pretty result by R. L. McFarland (cf. Theorem 3.3 of [Dil72]), $M$ can be decomposed into

$$
M=2^{-n} H_{n} \operatorname{diag}\left(\left\langle\xi, \ell_{0}\right\rangle, \cdots,\left\langle\xi, \ell_{2^{n}-1}\right\rangle\right) H_{n}
$$

where $\ell_{i}$ is the $i$ th row of $H_{n}$, a Sylvester-Hadamard matrix of order $2^{n}$.

Clearly

$$
M M^{T}=2^{-n} H_{n} \operatorname{diag}\left(\left\langle\xi, \ell_{0}\right\rangle^{2}, \cdots,\left\langle\xi, \ell_{2^{n}-1}\right\rangle^{2}\right) H_{n} .
$$

On the other hand, we always have

$$
M M^{T}=\left(\Delta\left(\alpha_{i} \oplus \alpha_{j}\right)\right),
$$

where $i, j=0,1, \ldots, 2^{n}-1$.

Comparing the two sides of (3), we have

$$
\left(\Delta\left(\alpha_{0}\right), \Delta\left(\alpha_{1}\right), \ldots, \Delta\left(\alpha_{2^{n}-1}\right)\right)=2^{-n}\left(\left\langle\xi, \ell_{0}\right\rangle^{2}, \ldots,\left\langle\xi, \ell_{2^{n}-1}\right\rangle^{2}\right) H_{n} .
$$

Equivalently we write

$$
\left(\Delta\left(\alpha_{0}\right), \Delta\left(\alpha_{1}\right), \ldots, \Delta\left(\alpha_{2^{n}-1}\right)\right) H_{n}=\left(\left\langle\xi, \ell_{0}\right\rangle^{2}, \ldots,\left\langle\xi, \ell_{2^{n}-1}\right\rangle^{2}\right) .
$$

In engineering, (4) is better known as (a special form of) the Wiener-Khintchine Theorem [Bea84]. A closely related result is Parseval's equation (Corollary 3, p. 416 of [MS77])

$$
\sum_{j=0}^{2^{n}-1}\left\langle\xi, \ell_{j}\right\rangle^{2}=2^{2 n}
$$

which also holds for any function $f$ on $V_{n}$.

Let $S$ be a set of vectors in $V_{n}$. The rank of $S$ is the maximum number of linearly independent vectors in $S$. Note that when $S$ forms a linear sub-space of $V_{n}$, its rank coincides with its dimension.

The distance between two functions $f_{1}$ and $f_{2}$ on $V_{n}$ can be expressed as $d\left(f_{1}, f_{2}\right)=2^{n-1}-\frac{1}{2}\left\langle\xi_{1}, \xi_{2}\right\rangle$, where $\xi_{1}$ and $\xi_{2}$ are the sequences of $f_{1}$ and $f_{2}$ respectively. (For a proof see for instance Lemma 6 of [SZZ94a].) Immediately we have:

Lemma 6. The nonlinearity of a function $f$ on $V_{n}$ can be calculated by

$$
N_{f}=2^{n-1}-\frac{1}{2} \max \left\{\left|\left\langle\xi, \ell_{i}\right\rangle\right|, 0 \leq i \leq 2^{n}-1\right\}
$$

where $\xi$ is the sequence of $f$ and $\ell_{0}, \ldots, \ell_{2^{n}-1}$ are the rows of $H_{n}$, namely, the sequences of the linear functions on $V_{n}$. 
The next lemma regarding splitting the power of 2 can be found in [SZZ94d]

Lemma 7. Let $n \geq 2$ be a positive integer and $p^{2}+q^{2}=2^{n}$ where both $p \geq 0$ and $q \geq 0$ are integers. Then $p=2^{\frac{1}{2} n}$ and $q=0$ when $n$ is even, and $p=q=2^{\frac{1}{2}(n-1)}$ when $n$ is odd.

In the next section we examine the distribution of the vectors in $\Re$.

\section{Distribution of $\Re$}

Let $f$ be a function on $V_{n}$. Assume that $f$ satisfies the propagation criterion with respect to all but a subset $\Re$ of $V_{n}$. Note that $\Re$ always contains the zero vector 0 . Write $\Re=\left\{0, \gamma_{1}, \ldots, \gamma_{s}\right\}$. Thus $|\Re|=s+1$.

Set $\Re^{c}=V_{n}-\Re$. Then $f$ satisfies the propagation criterion with respect to all vectors in $\Re^{c}$.

Consider the set of vectors $\{0\} \cup \Re^{c}$. Then $\{0\}$ is a linear sub-space contained in $\{0\} \cup \Re^{c}$. When $\left|\{0\} \cup \Re^{c}\right|>1,\{0, \gamma\}$ is a linear sub-space for any nonzero vector in $\Re^{c}$. We are particularly interested in linear sub-spaces with the maximum dimension contained in $\{0\} \cup \Re^{c}$. For convenience, denote by $\rho$ the maximum dimension and by $W$ a linear sub-space in $\{0\} \cup \Re^{c}$ that achieves the maximum dimension.

Obviously, $f$ is bent if and only if $\rho=n$, and $f$ does not satisfy the propagation criterion with respect to any vector if and only if $\rho=0$. The case when $1 \leq \rho \leq n-1$ is especially interesting.

Now let $U$ be a complementary sub-space of $W$, namely $U \oplus W=V_{n}$. Then each vector $\gamma \in V_{n}$ can be uniquely expressed as $\gamma=\alpha \oplus \beta$, where $\alpha \in W$ and $\beta \in U$. As the dimension of $W$ is $\rho$, the dimension of $U$ is equal to $n-\rho$. Write $U=\left\{0, \beta_{1}, \ldots, \beta_{2^{n-\rho}-1}\right\}$.

Proposition 8. $\Re \cap W=\{0\}$ and $\Re \cap\left(W \oplus \beta_{j}\right) \neq \phi$, where $W \oplus \beta_{j}=\left\{\alpha \oplus \beta_{j} \mid \alpha \in\right.$ $W\}, j=1, \ldots, 2^{n-\rho}-1$.

Proof. $\Re \cap W=\{0\}$ follows from the fact that $W$ is a sub-space of $\{0\} \cup \Re^{c}$. Next we consider $\Re \cap\left(W \oplus \beta_{j}\right)$.

Clearly,

$$
V_{n}=W \cup\left(W \oplus \beta_{1}\right) \cup \cdots \cup\left(W \oplus \beta_{2^{n-\rho}-1}\right) .
$$

In addition,

for $j=1, \ldots, 2^{n-p}-1$, and

$$
W \cap\left(W \oplus \beta_{j}\right)=\phi
$$

$$
\left(W \oplus \beta_{j}\right) \cap\left(W \oplus \beta_{i}\right)=\phi
$$

for any $j \neq i$. Assume for contradiction that $\Re \cap\left(W \oplus \beta_{j_{0}}\right)=\phi$ for some $j_{0}$, $1 \leq j_{0} \leq 2^{n-\rho}-1$. Then we have $W \oplus \beta_{j_{0}} \subseteq \Re^{c}$. In this case $W \cup\left(W \oplus \beta_{j_{0}}\right)$ must form a sub-space of $V_{n}$. This contradicts the definition that $W$ is a linear sub-space with the maximum dimension in $\{0\} \cup \Re^{c}$. This completes the proof.

The next corollary follows directly from the above proposition.

Corollary 9. The size of $\Re$ satisfies $|\Re| \geq 2^{n-\rho}$ and hence the rank of $\Re$ is at least $n-\rho$, where $\rho$ is the maximum dimension a linear sub-space in $\{0\} \cup \Re^{c}$ can achieve. 


\section{$5 \quad$ Relating Nonlinearity to Propagation Characteristics}

We proceed to the discussion of the nonlinearity of $f$. The main difficulty lies in finding a good approximation of $\left\langle\xi, \ell_{i}\right\rangle$ for each $i=0, \ldots, 2^{n}-1$, where $\xi$ is the sequence of $f$ and $\xi_{i}$ is a row of $H_{n}$.

First we assume that

$$
\begin{aligned}
W & =\left\{\gamma \mid \gamma=\left(a_{1}, \ldots, a_{\rho}, 0, \ldots, 0\right), a_{i} \in G F(2)\right\} \\
U & =\left\{\gamma \mid \gamma=\left(0, \ldots, 0, a_{\rho+1}, \ldots, a_{n}\right), a_{i} \in G F(2)\right\}
\end{aligned}
$$

where $W$ is a linear sub-space in $\{0\} \cup \Re^{e}$ that achieves the maximum dimension $\rho$ and $U$ is a complementary sub-space of $W$. The more general case where (5) or (6) is not satisfied can be dealt with after employing a nonsingular transform on the input of $f$. This will be discussed in the later part of this section.

Recall that $\Re=\left\{0, \gamma_{1}, \ldots, \gamma_{s}\right\}$ and $\Delta(\alpha)=\langle\xi(0), \xi(\alpha)\rangle$, where $\xi(\alpha)$ is the sequence of $f(x \oplus \alpha)$. Since $\Delta(\gamma) \neq 0$ for each $\gamma \in \Re$ while $\Delta(\gamma)=0$ for each $\gamma \in \Re^{c}=V_{n}-\Re$, (4) is specialized as

$$
\left(\Delta(0), \Delta\left(\gamma_{1}\right), \ldots, \Delta\left(\gamma_{s}\right)\right) Q=\left(\left\langle\xi, \ell_{0}\right\rangle^{2}, \ldots,\left\langle\xi, \ell_{2^{n}-1}\right\rangle^{2}\right)
$$

where $\xi$ is the sequence of $f, \ell_{i}$ is the $i$ th row of $H_{n}$ and $Q$ comprises the 0 th, $\gamma_{1}$ th $, \ldots, \gamma_{s}$ th rows of $H_{n}$. Note that $Q$ is an $(s+1) \times 2^{n}$ matrix.

Let $\ell$ be the $\gamma$ th row of $H_{n}$, where $\gamma \in \Re$. Note that $\gamma$ can be uniquely expressed as $\gamma=\alpha \oplus \beta$, where $\alpha \in W$ and $\beta \in U$. Let $\ell^{\prime}$ be the $\alpha$ th row of $H_{\rho}$ and $\ell^{\prime \prime}$ be the $\beta$ th row of $H_{n-\rho}$. As $H_{n}=H_{\rho} \times H_{n-\rho}$, $\ell$ can be represented by $\ell=\ell^{\prime} \times \ell^{\prime \prime}$, where $\times$ denotes the Kronecker product.

From the construction of $H_{n-\rho}$, we can see that the $\beta$ th row of $H_{n-\rho}$ is an all-one sequence of length $2^{n-\rho}$ if $\beta=0$, and a balanced $(1,-1)$-sequence of length $2^{n-\rho}$ if $\beta \neq 0$.

Recall that $\Re \cap W=\{0\}$ (see also Proposition 8). There are two cases associated with $\gamma=\alpha \oplus \beta \in \Re: \gamma=0$ and $\gamma \neq 0$. In the first case, $\ell=\ell^{\prime} \times \ell^{\prime \prime}$ is the all-one sequence of length $2^{n}$, while in the second case, we have $\beta \neq 0$ which implies that $\ell^{\prime \prime}$ is a balanced $(1,-1)$-sequence of length $2^{n-\rho}$ and hence $\ell=\ell^{\prime} \times \ell^{\prime \prime}$ is a concatenation of $2^{\rho}$ balanced $(1,-1)$-sequences of length $2^{n-\rho}$.

Therefore we can write $Q=\left(Q_{0}, Q_{1}, \ldots, Q_{2^{\rho}-1}\right)$, where each $Q_{i}$ is a $(1,-1)$ matrix of order $(s+1) \times 2^{n-p}$. It is important to note that the top row of each $Q_{i}$ is the all-one sequence, while the rest are balanced $(1,-1)$-sequences of length $2^{n-\rho}$.

With $Q_{0}$, we have

$$
\left(\Delta(0), \Delta\left(\gamma_{1}\right), \ldots, \Delta\left(\gamma_{s}\right)\right) Q_{0}=\left(\left\langle\xi, \ell_{0}\right\rangle^{2}, \ldots,\left\langle\xi, \ell_{2^{n-\rho}-1}\right\rangle^{2}\right) .
$$

Let $\sigma_{0}$ be the all-one sequence of length $2^{n-\rho}$. Then

$$
\left(\Delta(0), \Delta\left(\gamma_{1}\right), \ldots, \Delta\left(\gamma_{s}\right)\right) Q_{0} \sigma_{0}^{T}=\left(\left\langle\xi, \ell_{0}\right\rangle^{2}, \ldots,\left\langle\xi, \ell_{2^{n-\rho}-1}\right\rangle^{2}\right) \sigma_{0}^{T} .
$$

This causes

$$
\left(\Delta(0), \Delta\left(\gamma_{1}\right), \ldots, \Delta\left(\gamma_{s}\right)\right)\left[\begin{array}{c}
2^{n-\rho} \\
0 \\
\vdots \\
0
\end{array}\right]=\sum_{j=0}^{2^{n-\rho}-1}\left\langle\xi, \ell_{j}\right\rangle^{2}
$$


and

$$
\sum_{j=0}^{2^{n-\rho}-1}\left\langle\xi, \ell_{j}\right\rangle^{2}=2^{n-\rho} \Delta(0)=2^{n-\rho+n}=2^{2 n-\rho} .
$$

Similarly, with $Q_{i}, i=1, \ldots, 2^{\rho}-1$, we have

$$
\sum_{j=0}^{2^{n-\rho}-1}\left\langle\xi, \ell_{j+i 2^{n-\rho}}\right\rangle^{2}=2^{2 n-\rho} .
$$

Thus we have the following result:

Lemma 10. Assume that $f$, a function on $V_{n}$, satisfies the propagation criterion with respect to all but a subset $\Re$ of vectors in $V_{n}$. Set $\Re^{c}=V_{n}-\Re$ and let $W$ be a linear sub-space with the maximum dimension $\rho$, in $\{0\} \cup \Re^{c}$, and $U$ be a complementary sub-space of $W$. Assume that $W$ and $U$ satisfy (5) and (6) respectively. Then

$$
\sum_{j=0}^{2^{n-\rho}-1}\left\langle\xi, \ell_{j+i 2^{n-\rho}}\right\rangle^{2}=2^{2 n-\rho}
$$

for all $i=0,1, \ldots, 2^{\rho}-1$, where $\xi$ is the sequence of $f$ and each $\ell_{k}$ is a row of $H_{n}$.

Lemma 10 can be viewed as a refinement of Parseval's equation $\sum_{j=0}^{2^{n}-1}\left\langle\xi, \ell_{j}\right\rangle^{2}=$ $2^{2 n}$. It implies that $\left|\left\langle\xi, \ell_{j}\right\rangle\right| \leq 2^{n-\frac{1}{2} \rho}$ for all $j=0, \ldots, 2^{n}-1$. Therefore by Lemma 6 we have $N_{f} \geq 2^{n-1}-2^{n-\frac{1}{2} \rho-1}$.

So far we have assumed that $W$ and $U$ satisfy (5) and (6) respectively. When this is not the case, we can always find a nonsingular $n \times n$ matrix $A$ whose entries are from $G F(2)$ such that the sub-spaces $W^{\prime}$ and $U^{\prime}$ associated with $f^{\prime}(x)=f(x A)$ have the required forms. $f^{\prime}$ and $f$ have the same algebraic degree and nonlinearity (see Lemma 10 of [SZZ94b]). This shows that the following theorem is true.

Theorem 11. For any function on $V_{n}$, the nonlinearity of $f$ satisfies $N_{f} \geq$ $2^{n-1}-2^{n-\frac{1}{2} \rho-1}$, where $\rho$ is the maximum dimension of the linear sub-spaces in $\{0\} \cup \Re^{c}$.

Theorem 11 indicates that the nonlinearity of a function is determined by the maximum dimension that a linear sub-spaces in $\{0\} \cup \Re^{c}$ can achieve, but not by the size of $\Re^{c}$.

In [SZZ94e], we have proved that $N_{f} \geq 2^{n-1}-2^{\frac{1}{2}(n+t)-1}$, where $t$ is the rank of $\Re$. By Corollary 9, we have $t \geq n-\rho$. This implies that $2^{n-1}-2^{n-\frac{1}{2} \rho-1} \geq$ $2^{n-1}-2^{\frac{1}{2}(n+t)-1}$. Thus Theorem 11 is an improvement to the result in [SZZ94e]. This improvement can be demonstrated by a concrete example. In [SZZ94e], the following function on $V_{5}$

$$
\begin{aligned}
f_{5}\left(x_{1}, x_{2}, x_{3}, x_{4}, x_{5}\right)= & \left(1 \oplus x_{1}\right)\left(1 \oplus x_{2}\right) x_{3} \oplus\left(1 \oplus x_{1}\right) x_{2} x_{4} \oplus \\
& x_{1}\left(1 \oplus x_{2}\right)\left(x_{3} \oplus x_{4}\right) \oplus x_{1} x_{2}\left(x_{4} \oplus x_{5}\right)
\end{aligned}
$$


has been shown to satisfy the propagation criterion with respect to all but the following fives vectors in $V_{5}$ :

$$
\Re=\{(0,0,0,0,0),(0,0,0,0,1),(0,0,0,1,0),(0,0,1,0,0),(0,0,1,1,1)\} .
$$

The rank $t$ of $\Re$ is equal to 3 . By using the result of [SZZ94e], $N_{f_{5}} \geq 2^{5-1}-$ $2^{\frac{1}{2}(5+3)-1}=2^{4}-2^{3}=8$. On the other hand, we can set $W=\left\{\left(a_{1}, a_{2}, a_{3}, a_{4}, a_{5}\right) \mid a_{i} \in\right.$ $\left.G F(2), a_{1} \oplus a_{2} \oplus a_{3}=0\right\}$. $W$ is a four-dimensional sub-space in $\{0\} \cup \Re^{c}$. Using Theorem 11 with $\rho=4$, we have $N_{f_{5}} \geq 2^{5-1}-2^{5-\frac{1}{2} \rho-1}=2^{4}-2^{2}=12>8$. (Note that according to [CKHFMS85], the maximum nonlinearity a function on $V_{5}$ can achieve is 12 . Hence we have $N_{f_{5}}=12$.)

\section{A Tight Lower Bound on Nonlinearity of Functions with Propagation Characteristics}

By Theorem 11, $N_{f} \geq 2^{n-1}-2^{n-\frac{3}{2}}$ if $f$, a function on $V_{n}$, satisfies the propagation criterion with respect to at least one vector in $V_{n}$. This section shows that this lower bound can be significantly improved. Indeed we prove that $N_{f} \geq 2^{n-2}$ and also show that it is tight.

Theorem 12. If $f$, a function on $V_{n}$, satisfies the propagation criterion with respect to one or more vectors in $V_{n}$, then the nonlinearity of $f$ satisfies $N_{f} \geq$ $2^{n-2}$

Proof. As in the previous sections, we denote by $\Re$ the set of vectors in $V_{n}$ with respect to which the propagation criterion is not satisfied by $f$. We also let $\Re^{c}=V_{n}-\Re$, and $W$ be a linear sub-space in $\{0\} \cup \Re^{c}$ that achieves the maximum dimension $\rho$.

By Theorem 11, the theorem is trivially true when $\rho>1$. Next we consider the case when $\rho=1$. We prove this part by further refining the Parseval's equation.

As in the proof of Lemma 10, without loss of generality, we can assume that

$$
\begin{aligned}
W & =\left\{\gamma \mid \gamma=\left(a_{1}, 0, \ldots, 0\right), a_{1} \in G F(2)\right\} \\
U & =\left\{\gamma \mid \gamma=\left(0, a_{2}, \ldots, a_{n}\right), a_{i} \in G F(2)\right\}
\end{aligned}
$$

Similarly to Lemma 10 , we have

$$
\sum_{j=0}^{2^{n-1}-1}\left\langle\xi, \ell_{j+i 2^{2^{n-1}}}\right\rangle^{2}=2^{2 n-1}, i=0,1,
$$

where $\xi$ is the sequence of $f$ and $\ell_{k}$ is a row of $H_{n}$.

Comparing the first row of (2), we have

$$
\left(a_{0}, a_{1}, \ldots, a_{2^{n}-1}\right)=2^{-n}\left(\left\langle\xi, \ell_{0}\right\rangle, \cdots,\left\langle\xi, \ell_{2^{n}-1}\right\rangle\right) H_{n}
$$

or equivalently,

$$
2^{n}\left(a_{0}, a_{1}, \ldots, a_{2^{n}-1}\right)=\left(\left\langle\xi, \ell_{0}\right\rangle, \cdots,\left\langle\xi, \ell_{2^{n}-1}\right\rangle\right) H_{n}
$$


where each $a_{j}= \pm 1$ and $\left(a_{0}, a_{1}, \ldots, a_{2^{n}-1}\right)$ is the first row of the matrix $M$ described in (2).

Rewrite $\ell_{i}$, the $i$ th row of $H_{n}$, as $\ell\left(\alpha_{i}\right)$, where $\alpha_{i}$ is the binary representation of an integer $i$ in the ascending alphabetical order. Set

$$
N=\left(\left\langle\xi, \ell\left(\alpha_{i} \oplus \alpha_{j}\right)\right\rangle\right), 0 \leq i, j \leq 2^{n}-1
$$

$N$ is a symmetric matrix of order $2^{n}$ with integer entries. In [Rot76], Rothaus has shown that $N N=N N^{T}=2^{2 n} I_{2^{n}}$. We can split $N$ into four sub-matrices of equal size, namely

$$
N=\left[\begin{array}{ll}
N_{1} & N_{2} \\
N_{2} & N_{1}
\end{array}\right]
$$

where each $N_{j}$ is a matrix of order $2^{n-1}$. As $N N=2^{2 n} I_{2^{n}}$, we have $N_{1} N_{2}=0$.

Let $\left(c\left(\alpha_{0}\right), c\left(\alpha_{1}\right), \ldots, c\left(\alpha_{2^{n-1}-1}\right)\right)$ be an arbitrary linear sequence of length $2^{n-1}$. Then

$$
\left(c\left(\alpha_{0}\right), c\left(\alpha_{1}\right), \ldots, c\left(\alpha_{2^{n-1}-1}\right), c\left(\alpha_{0}\right), c\left(\alpha_{1}\right), \ldots, c\left(\alpha_{2^{n-1}-1}\right)\right)
$$

is a linear sequence of length $2^{n}$, and hence a row of $H_{n}$. Thus from (11), we have

$$
\sum_{j=0}^{2^{n-1}-1} c\left(\alpha_{j}\right)\left\langle\xi, \ell\left(\alpha_{j}\right)\right\rangle+\sum_{j=0}^{2^{n-1}-1} c\left(\alpha_{j}\right)\left\langle\xi, \ell\left(\alpha_{j} \oplus 2^{n-1}\right)\right\rangle= \pm 2^{n} .
$$

Hence

$$
\left(\sum_{j=0}^{2^{n-1}-1} c\left(\alpha_{j}\right)\left\langle\xi, \ell\left(\alpha_{j}\right)\right\rangle+\sum_{j=0}^{2^{n-1}-1} c\left(\alpha_{j}\right)\left\langle\xi, \ell\left(\alpha_{j} \oplus \alpha_{2^{n-1}}\right)\right\rangle\right)^{2}=2^{2 n}
$$

Rewrite the left hand side of (12) as

$$
\begin{aligned}
& \left(\sum_{j=0}^{2^{n-1}-1} c\left(\alpha_{j}\right)\left\langle\xi, \ell\left(\alpha_{j}\right)\right\rangle\right)^{2}+\left(\sum_{j=0}^{2^{n-1}-1} c\left(\alpha_{j}\right)\left\langle\xi, \ell\left(\alpha_{j} \oplus \alpha_{2^{n-1}}\right)\right\rangle\right)^{2} \\
+ & 2\left(\sum_{j=0}^{2^{n-1}-1} c\left(\alpha_{j}\right)\left\langle\xi, \ell\left(\alpha_{j}\right)\right\rangle\right)\left(\sum_{j=0}^{2^{n-1}-1} c\left(\alpha_{j}\right)\left\langle\xi, \ell\left(\alpha_{j} \oplus \alpha_{2^{n-1}}\right)\right\rangle\right)
\end{aligned}
$$

where

$$
\begin{aligned}
& \left(\sum_{j=0}^{2^{n-1}-1} c\left(\alpha_{j}\right)\left\langle\xi, \ell\left(\alpha_{j}\right)\right\rangle\right)\left(\sum_{j=0}^{2^{n-1}-1} c\left(\alpha_{j}\right)\left\langle\xi, \ell\left(\alpha_{j} \oplus \alpha_{2^{n-1}}\right)\right\rangle\right) \\
= & \sum_{t=0}^{2^{n-1}-1} \sum_{j=0}^{2^{n-1}-1} c\left(\alpha_{j}\right)\left\langle\xi, \ell\left(\alpha_{j}\right)\right\rangle c\left(\alpha_{j} \oplus \alpha_{t}\right)\left\langle\xi, \ell\left(\alpha_{j} \oplus \alpha_{t} \oplus \alpha_{2^{n-1}}\right)\right\rangle .
\end{aligned}
$$

As $\left(c\left(\alpha_{0}\right), c\left(\alpha_{1}\right), \ldots, c\left(\alpha_{2^{n-1}-1}\right)\right)$ is a linear sequence, $c\left(\alpha_{j}\right) c\left(\alpha_{j} \oplus \alpha_{t}\right)=c\left(\alpha_{t}\right)$. Hence $(13)$ can be written as

$$
\sum_{t=0}^{2^{n-1}-1} c\left(\alpha_{t}\right) \sum_{j=0}^{2^{n-1}-1}\left\langle\xi, \ell\left(\alpha_{j}\right)\right\rangle\left\langle\xi, \ell\left(\alpha_{j} \oplus \alpha_{t} \oplus \alpha_{2^{n-1}}\right)\right\rangle .
$$


Since $N_{1} N_{2}=0$,

$$
\sum_{j=0}^{2^{n-1}-1}\left\langle\xi, \ell\left(\alpha_{j}\right)\right\rangle\left\langle\xi, \ell\left(\alpha_{j} \oplus \alpha_{t} \oplus \alpha_{2^{n-1}}\right)\right\rangle=0 .
$$

This proves that (13) is equal to zero and hence

$$
\left(\sum_{j=0}^{2^{n-1}-1} c\left(\alpha_{j}\right)\left\langle\xi, \ell\left(\alpha_{j}\right)\right\rangle\right)^{2}+\left(\sum_{j=0}^{2^{n-1}-1} c\left(\alpha_{j}\right)\left\langle\xi, \ell\left(\alpha_{j} \oplus \alpha_{2^{n-1}}\right)\right\rangle\right)^{2}=2^{2 n} .
$$

By Lemma 7 ,

$$
\sum_{j=0}^{2^{n-1}-1} c\left(\alpha_{j}\right)\left\langle\xi, \ell\left(\alpha_{j}\right)\right\rangle=0 \text { or } \pm 2^{n}
$$

Since $\left(c\left(\alpha_{0}\right), c\left(\alpha_{1}\right), \ldots, c\left(\alpha_{2^{n-1}-1}\right)\right)$ is an arbitrary linear sequence of length $2^{n-1}$ and each linear sequence of length $2^{n-1}$ is a column of $H_{n-1}$, from (14) we have

$$
\left(\left\langle\xi, \ell_{0}\right\rangle, \ldots,\left\langle\xi, \ell_{2^{n}-1}\right\rangle\right) H_{n-1}=2^{n}\left(b_{0}, \ldots, b_{2^{n-1}-1}\right)
$$

where $b_{j}=0$ or \pm 1 . Therefore

$$
\left(\left\langle\xi, \ell_{0}\right\rangle, \ldots,\left\langle\xi, \ell_{2^{n}-1}\right\rangle\right) 2^{\frac{1}{2}(1-n)} H_{n-1}=2^{\frac{1}{2}(n+1)}\left(b_{0}, \ldots, b_{2^{n-1}-1}\right) .
$$

Recall that a matrix $A$ of order $s$ is said to be orthogonal if $A A^{T}=I_{s}$. It is easy to verify that $2^{\frac{1}{2}(1-n)} H_{n-1}$ is an orthogonal matrix. Thus

$$
\sum_{j=0}^{2^{n}-1}\left\langle\xi, \ell_{\alpha_{j}}\right\rangle^{2}=2^{n+1} \sum_{j=0}^{2^{n-1}-1} b_{j}^{2}
$$

On the other hand, by (10) we have

$$
\sum_{j=0}^{2^{n}-1}\left\langle\xi, \ell_{\alpha_{j}}\right\rangle^{2}=2^{2 n-1}
$$

Hence

$$
\sum_{j=0}^{2^{n-1}-1} b_{j}^{2}=\sum_{j=0}^{2^{n-1}-1}\left|b_{j}\right|=2^{n-2} .
$$

Now let $\sigma\left(\alpha_{i}\right)$ denote the $i$ th row of $H_{n-1}$, where $\alpha_{i} \in V_{n-1}$ is the binary representation of $i, i=0,1, \ldots, 2^{n-1}-1$. From (15),

$$
\left(\left\langle\xi, \ell_{0}\right\rangle, \cdots,\left\langle\xi, \ell_{2^{n}-1}\right\rangle\right) H_{n-1} \sigma\left(\alpha_{i}\right)^{T}=2^{n}\left(b_{0}, \ldots, b_{2^{n-1}-1}\right) \sigma\left(\alpha_{i}\right)^{T} .
$$

Note that

$$
\left\langle\sigma\left(\alpha_{i}\right), \sigma\left(\alpha_{j}\right)\right\rangle= \begin{cases}2^{n-1} & \text { if } j=i \\ 0 & \text { if } j \neq i\end{cases}
$$


Thus

$$
H_{n-1} \sigma\left(\alpha_{i}\right)^{T}=\left[\begin{array}{c}
0 \\
\vdots \\
0 \\
2^{n-1} \\
0 \\
\vdots \\
0
\end{array}\right]
$$

where $2^{n-1}$ is on the $i$ th position of the column vector.

Write $\sigma\left(\alpha_{i}\right)=\left(d_{0}, d_{1}, \ldots, d_{2^{n-1}-1}\right)$. Then

$$
\left(b_{0}, \ldots, b_{2^{n-1}-1}\right) \sigma\left(\alpha_{i}\right)^{T}=\sum_{j=0}^{2^{n-1}-1} d_{j} b_{j} .
$$

As $d_{j}= \pm 1$, we have

$$
\left|\sum_{j=0}^{2^{n-1}-1} d_{j} b_{j}\right| \leq \sum_{j=0}^{2^{n-1}-1}\left|b_{j}\right|=2^{n-2} .
$$

From (16), (17) and (18)

$$
2^{n-1}\left|\left\langle\xi, \ell_{i}\right\rangle\right| \leq 2^{n} \sum_{j=0}^{2^{n-1}-1}\left|b_{j}\right|=2^{2 n-2}
$$

and hence

$$
\left|\left\langle\xi, \ell_{i}\right\rangle\right| \leq 2^{n-1}
$$

where $i$ is an arbitrary integer in $\left[0, \ldots, 2^{n-1}-1\right]$. Similarly,

$$
\left|\left\langle\xi, \ell_{i}\right\rangle\right| \leq 2^{n-1}
$$

holds for all $i=2^{n-1}, 2^{n-1}+1, \ldots, 2^{n}-1$. By Lemma 6 , the nonlinearity of $f$ satisfies

$$
N_{f} \geq 2^{n-1}-2^{n-2}=2^{n-2} .
$$

This completes the proof.

As an immediate consequence, we have

Corollary 13. Let $f$ be a function on $V_{n}$. Then the following statements hold:

1. if the nonlinearity of $f$ satisfies $N_{f}<2^{n-2}$, then $f$ does not satisfy the propagation criterion with respect to any vector in $V_{n}$.

2. if $f$ satisfies the $S A C$, then the nonlinearity of $f$ satisfies $N_{f} \geq 2^{n-2}$. 
Finally we show that the lower bound $2^{n-2}$ is tight. We achieve the goal by demonstrating a function on $V_{n}$ whose nonlinearity is equal to $2^{n-2}$. Let $g\left(x_{1}, x_{2}\right)=x_{1} x_{2}$ be a function on $V_{2}$. Then the nonlinearity of $g$ is $N_{g}=1$. Now let $f\left(x_{1}, \ldots, x_{n}\right)=x_{1} x_{2}$ be a function on $V_{n}$. Then the nonlinearity of $f$ is $N_{f}=2^{n-2} N_{g}=2^{n-2}$ (see for instance Lemma 8 of [SZZ94c]). $f$ satisfies the propagation criterion with respect to all vectors in $V_{n}$ whose first two bits are nonzero, which count for three quarters of the vectors in $V_{n}$. It is not hard to verify that

$$
\{(0,0,0, \ldots, 0),(1,0,0, \ldots, 0),(0,1,0, \ldots, 0),(1,1,0, \ldots, 0)\}
$$

is the linear sub-space that achieves the maximum dimension $\rho=2$.

Thus we have a result described as follows:

Lemma 14. The lower bound $2^{n-2}$ as stated in Theorem 12 is tight.

\section{Conclusion}

We have shown quantitative relationships between nonlinearity, propagation characteristics and the SAC. A tight lower bound on the nonlinearity of a function with propagation characteristics is also presented.

This research has also introduced a number of interesting problems yet to be resolved. One of the problems is regarding the size and distribution of $\Re^{c}$, the set of vectors where the propagation criterion is satisfied by a function on $V_{n}$. For all the functions we know of, $\Re^{c}$ is either an empty set or a set with at least $2^{n-1}$ vectors. We believe that any further understanding of this problem will contribute to the research into the design and analysis of cryptographically strong nonlinear functions.

\section{Acknowledgments}

We would like to thank the anonymous referees for their helpful comments. The first author was supported in part by the Australian Research Council (ARC) under the reference numbers A49130102, A49131885 and A49232172, and by the Australian Telecommunications and Electronics Research Board (ATERB) under the reference number C010/058, the second author by ARC A49130102 and ATERB C010/058, and the third author by ARC A49232172 and ATERB N069/412. All authors were supported by a University of Wollongong Research Program grant.

This work was done while the third author was with the University of Wollongong.

\section{References}

[AT90] C. M. Adams and S. E. Tavares. Generating and counting binary bent sequences. IEEE Transactions on Information Theory, IT-36 No. 5:1170$1173,1990$. 
[Bea84] K. G. Beauchamp. Applications of Walsh and Related Functions with an Introduction to Sequency Functions. Microelectronics and Signal Processing. Academic Press, London, New York, Tokyo, 1984.

[BKPS93] L. Brown, M. Kwan, J. Pieprzyk, and J. Seberry. Improving resistance to differential cryptanalysis and the redesign of LOKI. In Advances in Cryptology - ASIACRYPT'91, Lecture Notes in Computer Science, vol. 739, pp. 6-50, Springer-Verlag, Berlin, New York, Tokyo, 1993.

[CKHFMS85] G. D. Cohen, M. G. Karpovsky, Jr. H. F. Mattson, and J. R. Schatz. Covering radius - survey and recent results. IEEE Transactions on Information Theory, IT-31(3):328-343, 1985.

[Dil72] J.F. Dillon. A survey of bent functions. The NSA Technical Journal, pp. 191-215, 1972. (unclassified).

[Eve88] J.-H. Evertse. Linear structures in blockciphers. In Advances in Cryptology - EUROCRYPT'87, Lecture Notes in Computer Science, vol. 304, pp. 249-266, Springer-Verlag, Berlin, Heidelberg, New York, 1988.

[Lai92] X. Lai. On the Design and Security of Block Ciphers. ETH Series in Information Processing. Hartung-Gorre Verlag Konstanz, Zürich, 1992.

[LaSM91] X. Lai and J. L. Massey ans S. Murphy. Markov ciphers and differential cryptanalysis. In D. W. Davies, editor, Advances in Cryptology - EUROCRYPT'91, Lecture Notes in Computer Science, vol. 547, pp. 17-38, Springer-Verlag, Berlin, New York, Tokyo, 1991.

[LM91] X. Lai and J. L. Massey. A proposal for a new block encryption standard. In I. B. Damgård, editor, Advances in Cryptology - EUROCRYPT'90, Lecture Notes in Computer Science, vol. 473, pp. 389-404, SpringerVerlag, Berlin, New York, Tokyo, 1991.

[Miy91] S. Miyaguchi. The FEAL cipher family. In Advances in Cryptology CRYPTO'90, Lecture Notes in Computer Science, vol. 537, pp. 627-638, Springer-Verlag, Berlin, New York, Tokyo, 1991.

[MS77] F. J. MacWilliams and N. J. A. Sloane. The Theory of Error-Correcting Codes. North-Holland, Amsterdam, New York, Oxford, 1977.

[MS90] W. Meier and O. Staffelbach. Nonlinearity criteria for cryptographic functions. In Advances in Cryptology - EUROCRYPT'89, Lecture Notes in Computer Science, vol. 434, pp. 549-562, Springer-Verlag, Berlin, Heidelberg, New York, 1990.

[NBS77] National Bureau of Standards. Data encryption standard. Federal Information Processing Standards Publication FIPS PUB 46, U.S. Department of Commerce, January 1977.

[NIST93] National Institute of Standards and Technology. Secure hash standard. Federal Information Processing Standards Publication FIPS PUB 180, U.S. Department of Commerce, May 1993.

[PGV91] B. Preneel, R. Govaerts, and J. Vandewalle. Boolean functions satisfying higher order propagation criteria. In Advances in Cryptology - EUROCRYPT'91, Lecture Notes in Computer Science, vol. 547, pp. 141152, Springer-Verlag, Berlin, Heidelberg, New York, 1991.

$\left[\mathrm{PLL}^{+} 91\right]$ B. Preneel, W. V. Leekwijck, L. V. Linden, R. Govaerts, and J. Vandewalle. Propagation characteristics of boolean functions. In Advances in Cryptology - EUROCRYPT'90, Lecture Notes in Computer Science, vol. 437, pp. 155-165, Springer-Verlag, Berlin, Heidelberg, New York, 1991.

[Riv92] R. Rivest. The MD5 message digest algorithm, April 1992. Request for Comments (RFC) 1321.

[Rot76] O. S. Rothaus. On "bent" functions. Journal of Combinatorial Theory, Ser. A, 20:300-305, 1976.

[SZZ94a] J. Seberry, X. M. Zhang, and Y. Zheng. Nonlinearity and propagation characteristics of balanced boolean functions. To appear in Information 
and Computation, 1994.

[SZZ94b] J. Seberry, X. M. Zhang, and Y. Zheng. Nonlinearly balanced boolean functions and their propagation characteristics. In Advances in Cryptology - CRYPTO'93, Lecture Notes in Computer Science, vol. 773, pp. 4960, Springer-Verlag, Berlin, Heidelberg, New York, 1994.

[SZZ94C] J. Seberry, X. M. Zhang, and Y. Zheng. On constructions and nonlinearity of correlation immune functions. In Advances in Cryptology - EUROCRYPT'93, Lecture Notes in Computer Science, vol. 765, pp. 181-199, Springer-Verlag, Berlin, Heidelberg, New York, 1994.

[SZZ94d] J. Seberry, X. M. Zhang, and Y. Zheng. Relationships among nonlinearity criteria. Presented at EUROCRYPT'94, 1994.

[SZZ94e] J. Seberry, X. M. Zhang, and Y. Zheng. Structures of cryptographic functions with strong avalanche characteristics. Presented at ASIACRYPT'94, 1994.

[Web85] A. F. Webster. Plaintext/ciphertext bit dependencies in cryptographic system. Master's Thesis, Department of Electrical Engineering, Queen's University, Ontario, Cannada, 1985.

[WT86] A. F. Webster and S. E. Tavares. On the design of S-boxes. In Advances in Cryptology - CRYPTO'85, Lecture Notes in Computer Science, vol. 219, pp. 523-534, Springer-Verlag, Berlin, Heidelberg, New York, 1986.

[YH89] R. Yarlagadda and J. E. Hershey. Analysis and synthesis of bent sequences. IEE Proceedings (Part E), 136:112-123, 1989.

[ZPS93] Y. Zheng, J. Pieprzyk, and J. Seberry. HAVAL - a one-way hashing algorithm with varialbe length of output. In Advances in Cryptology AUSCRYPT'92, Lecture Notes in Computer Science, vol. 718, pp. 83104, Springer-Verlag, Berlin, New York, Tokyo, 1993. 